\title{
Antimicrobial Resistance Genes in Salmonella enterica Isolates from Poultry and Swine in Thailand
}

\author{
Rungtip CHUANCHUEN ${ }^{1) *}$ and Pawin PADUNGTOD ${ }^{2)}$ \\ ${ }^{1)}$ Faculty of Veterinary Science, Chulalongkorn University, Bangkok 10330 and \\ ${ }^{2)}$ Faculty of Veterinary Medicine, Chiangmai University, Chiangmai 50100, Thailand
}

(Received 19 April 2009/Accepted 24 June 2009)

\begin{abstract}
A total of 184 resistant Salmonella enterica isolated from poultry and swine were investigated for the distribution of and associations between various antimicrobial resistance genes. All the isolates were screened for the presence of class 2 and 3 integrase genes and 18 resistance genes corresponding to their resistance phenotypes. None of the isolates carried class 2 and 3 integrons. The investigated resistance genes were responsible for resistance in $78 \%$ of the isolates. All the strains harboring more than one resistance gene were resistant to three or more antimicrobials. The $\operatorname{bla}_{\mathrm{TEM}}, \mathrm{cmlA}$, tetA, dfrA12, sul3, aadA1 genes were detected in the majority of strains resistant to ampicillin (87\%), chloramphenicol (63\%), tetracycline (60\%), trimethoprim (42\%), sulphonamides (42\%) and streptomycin/ spectinomycin $(61 \%)$, respectively. Two ciprofloxacin-resistant isolates had a single point mutation leading to Ser-83-Phe in GyrA or Thr-57-Ser in ParC. Statistical analysis revealed good correlation between the presence of antimicrobial resistance genes and corresponding resistance phenotype $(\mathrm{p}<0.01)$. The results indicated that the resistance genes play a major role in conferring resistance among the Salmonella isolates investigated.

KEY WORDS: antimicrobial resistance, resistance genes, Salmonella enterica.
\end{abstract}

Food-borne diseases caused by nontyphoid Salmonella enteric serovars represent an important public health problem worldwide $[24,30]$. In the last two decades, multidrug resistant (MDR) Salmonella isolates has been increasing and become a major health hazard [1, 3, 13]. These MDR Salmonella strains can be a direct threat to human health when their multidrug resistance phenotype interferes with the efficacy of antimicrobial treatment or an indirect threat when resistance is transferred to other human pathogens. The routine use of antimicrobials in food-producing animals for any purposes, including infection treatment, disease prevention and growth promotion has been a major factor in development and widespread dissemination of antimicrobial resistance in bacteria that are subsequently transferred to humans through the food chain. As a result, the European Union has banned the continuous feeding of antimicrobials used in human medicine to healthy animals for growth promotion purpose and recommended risk-based evaluations for all antimicrobial growth promoters [18].

In Thailand, antimicrobials have been extensively used in food animal production for decades. The country has faced the increasing-public health problem due to the misuse and overuse of these substances in food animals particularly MDR Salmonella that has been isolated along the food chain $[1,13,23]$. Use of antimicrobials in food animals has been regulated by the Thai Department of Livestock Development and all antimicrobials intended to use in food animals must be approved by Thai Food and Drug Administration. The relevant authorities in Thailand have attempted to rap-

\footnotetext{
* Correspondence to: Chuanchuen, R., Faculty of Veterinary Science, Chulalongkorn University, Bangkok 10330 Thailand. e-mail: rchuanchuen@yahoo.com
}

idly phase out antimicrobials as growth promoters and prohibited all antimicrobials being used as feed additives since 2005. Currently, the new regulatory policy for controlling use of antimicrobial feed additives is in the drafting process. In this case, manufacturers will be required to submit applications for evaluation and authorization of the current-existing and new feed additive antimicrobials for animals as growth promoter. Public health risk from development and dissemination of antimicrobial resistance in foodborne and human pathogens must be assessed and this scientific-based data must be readily available for pre-approval evaluation. Hence, surveillance of antimicrobial resistance in bacteria isolated from food animal reservoirs and study of mechanisms underlying the emergence and dissemination of antimicrobial resistance is crucially needed.

In our previous study, the prevalence, molecular characteristics and transferability of class 1 integrons in $S$. enterica from healthy swine and poultry were investigated [15]. In this study, we further characterized the molecular mechanisms of multidrug resistance among the Salmonella isolates. The objectives were to investigate distribution and associations of various resistance genes in MDR Salmonella isolates.

\section{MATERIALS AND METHODS}

Bacterial strains and antimicrobial susceptibility test: A total of 184 Salmonella isolates previously isolated were studied $[8,15]$. They were originated from poultry $(n=80)$ and swine $(n=104)$ and comprised of 34 serotypes including 24 Stanley, 19 Typhimurium, 15 Enteritidis, 13 Rissen, 13 Bsilla, 11 Weltevreden, 10 Salmonella subspecies I, 9 of each Anatum, Corvallis and Kedougou, 6 Albany, 5 
Amsterdam, 3 of each Altona, Agona, Orion, Virchow and Worthington, 2 of each Bovismorbifican, Give, Madjorio, Kentucky, Poona and Senftenberg, and another 10 serovars presented by only one isolate i.e. Bareilly, Blockley, Eppendorf, Kingston, Paratyphi B2, Panama, Schwarzengrund, Singapore, Thomson and Virginia. A hundred and forty four strains were obtained from the strain collection at National Institute of Animal Health (NIAH), Department of Livestock, Bangkok during 2003-2005. The other strains were isolated from samples (feces, rectal swabs, drinking water and feed) collected from healthy poultry and swine during 2005-2006. All of the Salmonella strains were isolated and tested for their biochemical characteristics using methods described in ISO6579:2002 (E) [14] and serotyped by slide agglutination using the Kauffmann-White serotyping schemes [25]. All the Salmonella isolates were subjected to antimicrobial susceptibility testing and the minimum inhibitory concentrations (MICs) were determined using a 2-fold agar dilution technique according to Clinical and Laboratory Standards Institute guidelines (CLSI) formerly NCCLS [20]. The antimicrobial agents tested and the breakpoints for clarifying Salmonella as resistant were as follows: ampicillin $(32 \mu \mathrm{g} / \mathrm{m} l)$, chlorampheni$\operatorname{col}(32 \mu \mathrm{g} / \mathrm{m} l)$, ciprofloxacin $(4 \mu \mathrm{g} / \mathrm{m} l)$, gentamicin $(8 \mu \mathrm{g} /$ $\mathrm{m} l)$, spectinomycin $(128 \mu \mathrm{g} / \mathrm{m} l)$, streptomycin $(32 \mu \mathrm{g} / \mathrm{m} l)$, sulfamethoxazole $(512 \mu \mathrm{g} / \mathrm{m} l)$, tetracycline $(16 \mu \mathrm{g} / \mathrm{m} l)$ and trimethoprim $(16 \mu \mathrm{g} / \mathrm{m} l)[11,21]$. Multidrug resistance was defined as isolates being resistant to 3 or more different classes of antimicrobials [22]. All the antimicrobials were purchased from Sigma-Aldrich (St. Louis, MO, U.S.A.).

All of the isolates used were previously classified as resistant to at least one antimicrobial agent [15]. Resistance rates to ampicillin, chloramphenicol, ciprofloxacin, gentamicin, spectinomycin, streptomycin, sulfamethoxazole, tetracycline and trimethoprim were $56 \%, 32 \%, 2 \%, 14 \%, 56 \%$, $69 \%, 76 \%, 66 \%$ and $38 \%$, respectively. Fifty-three percent of the isolates were resistant to 3 different classes of antimicrobials or more and considered multidrug resistance (MDR). Fifteen percent of the Salmonella strains carried class 1 integrons with resistance gene cassettes and 5\% of the isolates harbored SGI1-A or -F [15].

Polymerase Chain Reaction (PCR) and DNA sequencing: The 184 resistant isolates were screened for class 3 integrase, intI3 and 18 resistance genes corresponding to their resistance phenotypes using PCR. Ampicillin-resistant isolates $(n=103)$ were screened for the presence of the $b l a_{\mathrm{PSE}-1}$ and bla $a_{\text {TEM }}$ genes. Chloramphenicol-resistant isolates $(\mathrm{n}=59)$ were investigated for the catA, catB and $\mathrm{cmlA}$ genes. Gentamicin-resistant strains $(n=26)$ were screened for the $a a d B$ gene. All strains resistant to tetracycline $(n=86)$ were examined for the presence of the tet $A$ and tet $B$ genes. Trimethoprim-resistant isolates $(n=69)$ were investigated for the $d f r A 1, d f r A 10$ and $d f r A 12$ genes. Spectinomycin-resistant isolates $(n=103)$ were screened for the presence of the aadA1 and aadA2 genes. Strains resistant to streptomycin $(n=127)$ were investigated for the aadA1, aadA2, str $A$ and $s t r B$ genes. All the strains resistant to sulfamethoxazole $(n=139)$ were screened for the presence of the sul1, sul2 and sul3 genes.

For all ciprofloxacin-resistance isolates $(n=3)$, mutations in the quinolone resistance-determining regions (QRDRs) of the gyrA, gyrB, parC and parE genes were determined. The sequences were analyzed using the BLAST search available at the website of the National Center for Biotechnology Information. Two Salmonella isolates susceptible to ciprofloxacin were used as control.

Template DNA of all isolates was prepared by the whole cell boiled lysate procedure [16]. PCR was performed in a final volume of $25 \mu l$ using PCR master mix of Eppendorf ${ }^{\mathbb{R}}$ MasterMix (Eppendorf, Hamburg, Germany) according to the manufacturer's protocol in a PCR Sprint Thermocycler $^{\circledR}$ (Thermo Electron Corporation ${ }^{\circledR}$, Cambridge, UK). All primers used are listed in Table 1. The following thermal cycles were used to amplify all resistance genes and the QRDRs: one cycle at $94^{\circ} \mathrm{C}$ for $5 \mathrm{~min} ; 30$ cycles of $94^{\circ} \mathrm{C}$ for $45 \mathrm{~s}, 57^{\circ} \mathrm{C}$ for $45 \mathrm{~s}$, and extension at $72^{\circ} \mathrm{C}$ for $1 \mathrm{~s}$; and a final extension at $72^{\circ} \mathrm{C}$ for $5 \mathrm{~min}$. The int 13 gene was amplified using the same denaturation and annealing conditions, except that the extension time was changed to $45 \mathrm{sec}$. The representatives of PCR products were purified using the QIAQuick Gel Extraction kit (Qiagen, Hilden, Germany) and submitted for sequencing at Macrogen Inc. (Seoul, South Korea). Salmonella Paratyphi B var Java [28] and pAV3.5 [29] were used as positive controls for intI2 and intI3, respectively

Realtime PCR: All the isolates were screened for the presence of class 2 integrase gene, intI2, using realtime PCR. PCR reactions were carried out in a final volume of $25 \mu l$ using Biotools QuantiMix EASY SYG Kit (Biotools B\&M Labs S.A., Madrid, Spain) as described by the manufacturer in a Rotor-Gene 3000 Real Time Thermal Cycler (Corbett Research, Sydney, Australia). Amplification conditions were $50^{\circ} \mathrm{C}$ for $2 \mathrm{~min}, 95^{\circ} \mathrm{C}$ for $2 \mathrm{~min}, 40$ cycles of $95^{\circ} \mathrm{C}$ for $15 \mathrm{~s}, 60^{\circ} \mathrm{C}$ for $30 \mathrm{~s}$ followed by holding at $4^{\circ} \mathrm{C}$. Salmonella Paratyphi B var Java was used as positive control for intI2 [28]. The specificity of realtime PCR was confirmed by melting curve analysis and detection of a $247 \mathrm{bp}$ PCR-amplicon by gel electrophoresis.

Statistical analysis: All statistical analysis was carried out using STATA software Version 8.0 (STATA Corp., College Station, TX, U.S.A.). The significance level of association between having resistance genes and resistance phenotype was determined using Fisher's exact test. Comparison among median MIC level of isolates with different number of resistance gene (s) was performed using Kruskal-Wallis test.

\section{RESULTS}

No class 2 or 3 integrons were detected in the Salmonella isolates in this study. The distribution of the various resistance genes and the prevalence of the corresponding serovars are shown in Table 2 . The investigated resistance genes were responsible for resistance in $78 \%$ of the isolates 
Table 1. Primers used in this study

\begin{tabular}{|c|c|c|c|c|}
\hline $\begin{array}{l}\text { Target gene or } \\
\text { region }\end{array}$ & Primer & Sequence of primers $\left(5^{\prime}-3^{\prime}\right)$ & Size (bp) & Reference \\
\hline \multirow[t]{2}{*}{$\operatorname{aadA1}$} & aadA1-F & CTCCGCAGTGGATGGCGG & 631 & {$[8]$} \\
\hline & aadA1-R & GATCTGCGCGCGAGGCCA & & \\
\hline \multirow[t]{2}{*}{$\operatorname{aad} A 2$} & aadA2-F & CATTGAGCGCCATCTGGAAT & 500 & [8] \\
\hline & aadA2-R & ACATTTCGCTCATCGCCGGC & & \\
\hline \multirow[t]{2}{*}{$a a d B$} & aadB-F & CTAGCTGCGGCAGATGAGC & 300 & This study \\
\hline & aadB-R & CTCAGCCGCCTCTGGGCA & & \\
\hline \multirow[t]{2}{*}{$b l a_{\mathrm{PSE}-1}$} & bla $_{\mathrm{PSE} 1}-\mathrm{F}$ & GCAAGTAGGGCAGGCAATCA & 422 & [8] \\
\hline & bla $a_{P S E 1}-\mathrm{R}$ & GAGCTAGATAGATGCTCACAA & & \\
\hline \multirow[t]{2}{*}{$b^{b l a \mathrm{TEM}}$} & bla $_{\mathrm{TEM}}-\mathrm{F}$ & ATCAGTTGGGTGCACGAGTG & 608 & {$[8]$} \\
\hline & bla $_{\mathrm{TEM}}-\mathrm{R}$ & ACGCTCACCGGCTCCAGA & & \\
\hline \multirow[t]{2}{*}{ catA } & catA-F & CCAGACCGTTCAGCTGGATA & 454 & {$[8]$} \\
\hline & catA-R & CATCAGCACCTTGTCGCCT & & \\
\hline \multirow[t]{2}{*}{$c a t B$} & catB-F & CGGATTCAGCCTGACCACC & 461 & [8] \\
\hline & catB-R & ATACGCGGTCACCTTCCTG & & \\
\hline \multirow[t]{2}{*}{ cmlA } & $\mathrm{cmlA}-\mathrm{F}$ & TGGACCGCTATCGGACCG & 641 & [7] \\
\hline & cmlA-B & CGCAAGACACTTGGGCTGC & & \\
\hline \multirow[t]{2}{*}{ dfrAl } & dfrA1-F & CAATGGCTGTTGGTTGGAC & 254 & {$[8]$} \\
\hline & dfrA1-R & CCGGCTCGATGTCTATTGT & & \\
\hline \multirow[t]{2}{*}{ dfralo } & dfrA10-F & TCAAGGCAAATTACCTTGGC & 432 & This sudy \\
\hline & dfrA10-R & ATCTATTGGATCACCTACCC & & \\
\hline \multirow[t]{2}{*}{ dfrA12 } & dfrA12-F & TTCGCAGACTCACTGAGGG & 330 & {$[8]$} \\
\hline & dfrA12-R & CGGTTGAGACAAGCTCGAAT & & \\
\hline \multirow[t]{2}{*}{ strA } & strA-F & TGGCAGGAGGAACAGGAGG & 405 & This study \\
\hline & strA-R & AGGTCGATCAGACCCGTGC & & \\
\hline \multirow[t]{2}{*}{$\operatorname{str} B$} & strB-F & GCGGACACCTTTTCCAGCCT & 621 & This study \\
\hline & strB-R & TCCGCCATCTGTGCAATGCG & & \\
\hline \multirow[t]{2}{*}{ sul1 } & sul1-F & CGGACGCGAGGCCTGTATC & 591 & {$[6]$} \\
\hline & sull-R & GGGTGCGGACGTAGTCAGC & & \\
\hline \multirow[t]{2}{*}{ sul2 } & sul2-F & GCGCAGGCGCGTAAGCTGAT & 514 & This study \\
\hline & sul2-R & CGAAGCGCAGCCGCAATTC & & \\
\hline \multirow[t]{2}{*}{ sul3 } & sul3-F & GGGAGCCGCTTCCAGTAAT & 500 & [7] \\
\hline & sul3-R & TCCGTGACACTGCAATCATTA & & \\
\hline \multirow[t]{2}{*}{ tetA } & tetA-F & GCTGTCGGATCGTTTCGG & 658 & [8] \\
\hline & tetA-R & CATTCCGAGCATGAGTGCC & & \\
\hline \multirow[t]{2}{*}{ tetB } & tetB-F & CTGTCGCGGCATCGGTCAT & 615 & {$[8]$} \\
\hline & tetB-R & CAGGTAAAGCGATCCCACC & & \\
\hline \multirow[t]{2}{*}{ intI2 } & intI2 & GGCAGACAGTTGCAAGACAA & 247 & This study \\
\hline & intI2 & AAGCGATTTTCTGCGTGTTT & & \\
\hline \multirow[t]{2}{*}{ intI3 } & intI3-F & CCGGTTCAGTCTTTCCTCAA & 155 & This study \\
\hline & intI3-R & GAGGCGTGTACTTGCCTCAT & & \\
\hline \multirow[t]{2}{*}{ QRDR of $g y r A$} & gyrA-F & GCTGAAGAGCTCCTATCTGG & 436 & This study \\
\hline & gyrA-R & GGTCGGCATGACGTCCGG & & \\
\hline \multirow[t]{2}{*}{ QRDR of $g y r B$} & gyrB-F & GCGCGCTCGATTTAGCCG & 287 & This study \\
\hline & gyrB-R & TGATAGCGCAGCTTGTCCG & & \\
\hline QRDR of parC & parC-F & GTACGTGATCATGGATCGTG & 390 & This study \\
\hline & parC-R & TTCCTGCATGGTGCCGTCG & & \\
\hline QRDR of parE & parE-F & GCGATCGCGAATATCAGGCG & 364 & This study \\
\hline & parE-R & CAGTTGTTCCAGTACGCCC & & \\
\hline
\end{tabular}

and the existence of each resistance gene did not appear to be specific to a particular serovar. Most of ampicillin-resistant strains $(93 \%)$ contained either $b l a_{\mathrm{PSE}-1}$ and/or $b l a_{\mathrm{TEM}}$, of which the $b l a_{\text {TEM }}$ gene was the most commonly-identified genes $(87 \%)$. The majority of chloramphenicol- $(63 \%)$ and gentamicin- $(88 \%)$ resistant isolates carried $\mathrm{cmlA}$ and $\mathrm{aadB}$ genes respectively. The tet $A$ gene was detected in the majority of tetracycline-resistant strains $(60 \%)$ and the dfrA12 gene was most prevalent among trimethoprim-resistant strains (42\%). Most of sulphonamide-resistant strains (76\%) contained at least one sul gene tested, of which the sul3 gene was the most frequently-detected genes (42\%). Resistance to spectinomycin and streptomycin was mediated by aadAl in the majority of the spectinomycin- $(68 \%)$ 
Table 2. Prevalence of resistance genes in relation to resistance phenotypes and distribution of $S$. enterica serovar from poultry ( $n=84)$ and swine $(n=104)$

\begin{tabular}{|c|c|c|c|c|}
\hline \multirow{2}{*}{$\begin{array}{l}\text { Resistance phenotype } \\
(n)\end{array}$} & \multirow[t]{2}{*}{ Resistance genes } & \multicolumn{2}{|c|}{ No. of isolates $(\%)^{a)}$} & \multirow[t]{2}{*}{ Serovar ${ }^{b)}(\text { no. of isolates })^{c)}$} \\
\hline & & Poultry & swine & \\
\hline \multirow[t]{4}{*}{ Ampicillin (103) } & $b l a_{\mathrm{PSE} 1}$ & $4(5)$ & $2(2)$ & $\operatorname{Alb}(5), \operatorname{Kin}(1)$ \\
\hline & $b l a \mathrm{TEM}$ & $19(23)$ & $42(40)$ & $\begin{array}{l}\text { Alt, Ana(2), Bov, Bsi(10), Cor(2), Eme, Ent (6), Giv, } \\
\operatorname{Ked}(6), \operatorname{Ken}(2), \operatorname{Ori}(2), \operatorname{Ris}(5), \operatorname{Sen}(2), \operatorname{Sta}(8), \operatorname{subI}(3), \text { Tho, } \\
\text { Typ(2), Wel (3), Wor(2) }\end{array}$ \\
\hline & both & $13(15)$ & $16(15)$ & Alt, Epp, Ked, Sch, Sta (12), subI(4),Typ (8), Wel \\
\hline & at least one & $36(43)$ & $60(58)$ & All inclusive strains \\
\hline \multirow[t]{2}{*}{ Chloramphenicol (59) } & catB & $0(0)$ & $1(1)$ & subI \\
\hline & cmlA & $7(8)$ & $30(29)$ & $\operatorname{Alt}(2), \operatorname{Bsi}(8)$, Giv, subI(3), Ked(8), Sta(14), Wel \\
\hline Gentamicin (26) & $a a d B$ & $7(8)$ & $16(15)$ & Ana, Epp, Ked(9), Ken, Sch, subI(5), Sta, Typ(3), Wel \\
\hline \multirow[t]{4}{*}{ Spectinomycin (103) } & aadAl & $14(17)$ & $33(32)$ & $\begin{array}{l}\text { Alb, Alt(2), Bsi(12), Bov, Cor, Eme, Ent, Ken, Par, Poo, Ris(4), } \\
\text { Sch, Sen, Sta(11), subI(4), Typ, Vic(2), Wel }\end{array}$ \\
\hline & aadA2 & $0(0)$ & $2(2)$ & $\operatorname{Sta}(2)$ \\
\hline & both & $6(7)$ & $17(16)$ & Ana, Epp, Giv, Ked(8), Ris, Sta(4), $\operatorname{subI}(3), \operatorname{Typ}(3)$, Wel \\
\hline & at least one & $20(24)$ & $52(50)$ & All inclusive strains \\
\hline \multirow[t]{7}{*}{ Streptomycin (127) } & $\operatorname{aadA1}$ & $9(11)$ & $8(8)$ & Alb, Eme, Par, Ris(3), Sen, Sta, subI(3), Typ(5), Vic \\
\hline & strA, strB & $9(11)$ & $12(12)$ & $\begin{array}{l}\text { Alt, Bsi, Cor(5), Ken, Ori, Poo, Ris(3), Sen, Sta, Tho, Wel(2), } \\
\text { Wor(3) }\end{array}$ \\
\hline & aadA1, aadA2 & $2(2)$ & $8(8)$ & $\operatorname{Ked}(4), \operatorname{Ris}, \operatorname{subI}(2), \operatorname{Typ}(2)$, Wel \\
\hline & strA, strB, aadAl & $7(8)$ & $30(29)$ & Alt(2), Bov, Bsi(12), Cor, Poo, Ris(2), Sta(14),Typ(2), Vic, Wel \\
\hline & strA, strB, aadA2 & $0(0)$ & $2(2)$ & $\operatorname{Sta}(2)$ \\
\hline & strA, strB, aadA1, aadA2 & $3(4)$ & $3(3)$ & Giv, subI, $\operatorname{Sta}(4)$ \\
\hline & at least one & $30(36)$ & $63(61)$ & All inclusive strains \\
\hline \multirow[t]{8}{*}{ Sulfamethoxazole (139) } & sull & $10(12)$ & $8(8)$ & $\begin{array}{l}\text { Alb(5), Ana, Eme(2),Kin, Mad, Ori, Par, Ris, Sch, subI(2), } \\
\text { Typ, Wel }\end{array}$ \\
\hline & sul2 & $8(10)$ & $9(9)$ & Ago, Cor(6), Ent, Ori, Ris, Sta, Typ(6) \\
\hline & sul3 & $7(8)$ & $30(29)$ & $\begin{array}{l}\text { Alt(3), Ana(3), Bov, Bsi(11), Ent, Ked(6), Sta(5), Tho, Typ(3), } \\
\text { Wel(2), Wor }\end{array}$ \\
\hline & all three & $2(2)$ & $7(7)$ & Ked, Sta, subI(5), Typ, Wel \\
\hline & sull, sul2 & $0(0)$ & $2(2)$ & $\operatorname{Typ}(2)$ \\
\hline & sull,sul3 & $9(11)$ & $5(5)$ & Giv, Epp, Ked(2), Ken, Ris, Sta(6), subI, Wel \\
\hline & sul2,sul3 & $0(0)$ & $8(8)$ & Bsi(2), Sta(5), Wel \\
\hline & at least one & $36(43)$ & $69(66)$ & All inclusive strains \\
\hline \multirow[t]{4}{*}{ Tetracycline (122) } & tetA & $13(15)$ & $47(45)$ & $\begin{array}{l}\text { Ago, Alb, Ana(3), Blo, Bov, Bsi(5), Cor(3), Epp, Giv, } \\
\text { Ked(5), Ken, } \\
\text { Mad, Ris(7), Sch, Sta(15), } \operatorname{subI(5),~Typ(5),~Wel(2),~Wor~}\end{array}$ \\
\hline & tet $B$ & $1(1)$ & $4(4)$ & Ori, Typ(3), Vig \\
\hline & both & $6(7)$ & $8(8)$ & Par, Ked(4), Sta(4), subI, Typ(3), Wel \\
\hline & at least one & $20(24)$ & $59(57)$ & All inclusive strains \\
\hline \multirow[t]{5}{*}{ Trimethoprim (69) } & dfrAl & $6(7)$ & $1(1)$ & $\operatorname{Alb}(5)$, Eme, Mad \\
\hline & dfrA10 & $1(1)$ & $1(1)$ & Kin, Wel \\
\hline & dfrA12 & $9(11)$ & $19(18)$ & $\begin{array}{l}\text { Ana, Giv, Ken,Par, Ris(3), Sch, Sta(8), } \operatorname{subI}(4) \text {, Typ(5), } \\
\text { Wel, Wor(2) }\end{array}$ \\
\hline & dfrA10, dfrA12 & $0(0)$ & $1(1)$ & subI \\
\hline & at least one & $16(19)$ & $22(21)$ & All inclusive strains \\
\hline
\end{tabular}

a) Some of bla $\mathrm{PSE}_{1}(2,2 \%)$, aadA2 $(18,17 \%), d f r A 1(8,12 \%)$ and $d f r A 12(16,23 \%)$ were the inserted gene cassettes in class 1 integrons as previously observed [13].

b) Abbreviations: Alb, Albany; Alt, Altona; Ago, Agona; Ams, Amsterdam; Ana, Anatum; Bar, Bareilly; Bov, Bovismorbifican; Bsi, Bsilla; Blo, Blockley; Cor, Corvallis; Ent, Enteritidis; Eme, Emek; Epp, Eppendorf; Giv, Give; Ked, Kedougou; Kin, Kingston; Ken, Kentucky; Mad, Madjorio; Ori, Orion; Pan, Panama; Par, Paratyphi B2; Poo, Poona; Ris, Rissen; Sta, Stanley; subI, subspecies I; Sch, Schwarzengrund; Sen, Senftenberg; Sin, Singapore; Typ, Typhimurium; Tho, Thomson; Vig, Virginia; Vic, Virchow; Wor, Worthington; and Wel, Weltevreden.

c) Serovars represented by only one isolate are shown without number.

and streptomycin- $(56 \%)$ resistant isolates. The presence of different genes within the same strains, encoding resistance to the same antimicrobials, was detected in 53\%.

Sequence analysis of the QRDRs of the gyrA, gyrB, parC, and parE genes revealed that only 2 isolates harbored a point mutation. Replacement of $\mathrm{C} 248$ with $\mathrm{G}$ leading to a Ser-83-Phe substitution in GyrA was identified in a serovar Senftenberg isolate and a point mutation C-170-G leading to 
Table 3. Distribution of resistance genes in relation to antimicrobial resistance patterns $(n=184)$

\begin{tabular}{|c|c|c|}
\hline Antibiotic resistance pattern ${ }^{a}$ & $\begin{array}{l}\text { No. of } \\
\text { isolates }(\%)\end{array}$ & Resistance gene (No.) \\
\hline SPC & $3(1.6)$ & - \\
\hline STR & $4(2.2)$ & $\operatorname{str} A(1), \operatorname{str} B(1)$ \\
\hline SUL & $9(4.9)$ & $\operatorname{sul} 2(1)$ \\
\hline TET & $6(3.3)$ & $\operatorname{tet} A(1)$ \\
\hline AMP, STR & $4(2.2)$ & $b l a_{\mathrm{TEM}}(1), \operatorname{str} A(2), \operatorname{str} B(2)$ \\
\hline AMP, SUL & $3(1.6)$ & $\operatorname{bla}_{\mathrm{TEM}}(2), \operatorname{sul} 3(1)$ \\
\hline SPC, TET & $2(1.1)$ & $\operatorname{tet} A(1)$ \\
\hline STR, SUL & $4(2.2)$ & $\operatorname{aadAl}(1), \operatorname{str} \mathrm{A}(2), \operatorname{str} B(2), \operatorname{sul} 2(1)$ \\
\hline STR, TET & $5(2.7)$ & $\operatorname{aadA1}(1), \operatorname{str} A(2), \operatorname{str} B(2), \operatorname{tet} A(1), \operatorname{tet} B(1)$ \\
\hline AMP, SPC, SUL & $5(2.7)$ & $\operatorname{aadAl}(1), \operatorname{bla}_{\mathrm{PSE}-1}(1), \operatorname{bla}_{\mathrm{TEM}}(4), \operatorname{sul} 3(3)$ \\
\hline SPC, STR, SUL & $8(4.3)$ & aadAl(4), dfrAl(1), strA(3), strB(3), sull(3), sul2(1), sul3(4) \\
\hline SPC, STR, TET & $5(2.7)$ & $\operatorname{aadAl}(4), \operatorname{str} A(4), \operatorname{str} B(4), \operatorname{tet} A(1)$ \\
\hline STR, SUL, TET & $4(2.2)$ & $\operatorname{str} A(3), \operatorname{str} B(3), \operatorname{sul} 2(4), \operatorname{tet} A(3)$ \\
\hline SUL, TET, TRI & $5(2.7)$ & dfrA12(1), sul2(4), tetA(3), tetB(3) \\
\hline AMP, TET, STR & $2(1.1)$ & $\operatorname{aadAl}(1), \operatorname{bla} a_{\mathrm{PE}-1}(2), \operatorname{bla} a_{\mathrm{TEM}}(2), \operatorname{str} A(2), \operatorname{str} B(2), \operatorname{tet} A(1)$ \\
\hline AMP, STR, SUL & $3(1.6)$ & $\operatorname{aadAl}(2), \operatorname{bla}_{\mathrm{PSE}-1}(2), \operatorname{bla}_{\mathrm{TEM}}(3), \operatorname{str} A(1), \operatorname{str} B(1), \operatorname{sul} 3(3)$ \\
\hline AMP, STR, SUL, TET & $4(2.2)$ & $\operatorname{aadAl}(1), \operatorname{bla}_{\mathrm{TEM}}(4), \operatorname{str} A(2), \operatorname{str} B(2), \operatorname{sul} 2(2), \operatorname{sul3}(3)$ \\
\hline AMP, SPC, SUL, TET & $2(1.1)$ & $\operatorname{bla}_{\mathrm{TEM}}(1), \operatorname{sul} 3(2), \operatorname{tet} A(1)$ \\
\hline AMP, SPC, STR, SUL & $2(1.1)$ & $\operatorname{aadA1}(2), \operatorname{bla}_{\mathrm{TEM}}(2), \operatorname{str} A(1), \operatorname{str} B(1), \operatorname{sul} 2(1), \operatorname{sul} 3(2)$ \\
\hline CHP, SUL, TET, TRI & $2(1.1)$ & - \\
\hline CHP, SPC, STR, SUL & $2(1.1)$ & $\operatorname{aadAl}(1), \operatorname{cml} A(1), \operatorname{str} A(1), \operatorname{str} B(1), \operatorname{sul} 3(1)$ \\
\hline SPC, STR, SUL, TRI & $2(1.1)$ & $\operatorname{aadAl}(1), \operatorname{dfrAl}(1), d f r A 10(1), d f r A 12(1), \operatorname{str} A(1), \operatorname{str} B(1), \operatorname{sull}(2), \operatorname{sul2}(1), \operatorname{sul} 3(1)$ \\
\hline SPC, STR, SUL, TET & $4(2.2)$ & $\operatorname{aadAl}(1), \operatorname{str} A(2), \operatorname{str} B(2), \operatorname{sul}(2), \operatorname{tet} A(1)$ \\
\hline AMP, SPC, STR, SUL, TET & $4(2.2)$ & 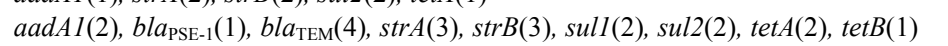 \\
\hline SPC, STR, SUL, TET, TRI & $3(1.6)$ & $\operatorname{aadAl}(2), \operatorname{dfr} A 10(1), \operatorname{dfr} A 12(2), \operatorname{sull}(3), \operatorname{tet} A(3), \operatorname{tet} B(1)$ \\
\hline AMP, CHP, SPC, STR, SUL, TET & $12(6.5)$ & $\begin{array}{l}\operatorname{aadA1}(10), \operatorname{aad} A 2(4), \operatorname{bla}_{\mathrm{PSE}-1}(5), \operatorname{bla}_{\mathrm{TEM}}(12), \operatorname{cml} A(12), \operatorname{str} A(11), \operatorname{str} B(11), \\
\operatorname{sul3}(12), \operatorname{tet} A(9)\end{array}$ \\
\hline AMP, CHP, STR, SUL, TET, TRI & $4(2.2)$ & $\begin{array}{l}\operatorname{aadA1}(1), \operatorname{bla}_{\mathrm{PSE}-1}(3), \operatorname{bla}_{\mathrm{TEM}}(1), \operatorname{cml}(1), \operatorname{dfr} A 1(3), \operatorname{str} A(1), \operatorname{str} B(1), \operatorname{sull}(3), \\
\operatorname{sul2}(1), \operatorname{sul}(1), \operatorname{tet} A(1)\end{array}$ \\
\hline AMP, SPC, STR, SUL, TET, TRI & $6(3.3)$ & $\begin{array}{l}\text { aadAl(4), aadA2(1), } \operatorname{bla}_{\mathrm{PSE}-1}(2), \operatorname{bla}_{\mathrm{TEM}}(6), \operatorname{dfr} A 12(4), \operatorname{str} A(6), \operatorname{str} B(6), \operatorname{sull}(5), \\
\operatorname{sul}(2), \operatorname{sul}(4), \operatorname{tet} A(1)\end{array}$ \\
\hline AMP, CHP, SPC, STR, TET, TRI & $2(1.1)$ & 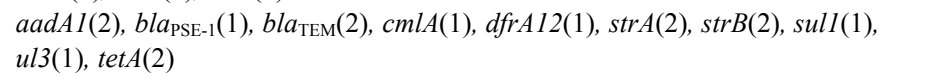 \\
\hline AMP, GEN, STR, SUL, TET, TRI & $3(1.6)$ & $\operatorname{aadAl}(2), \operatorname{aadB}(3), \operatorname{bla}_{\mathrm{PSE}-1}(2), \operatorname{bla} a_{\mathrm{TEM}}(3), \operatorname{str} A(1), \operatorname{str} B(1), \operatorname{sul}(1), \operatorname{tet} B(1)$ \\
\hline AMP, GEN, SPC, SUL, TET, TRI & $3(1.6)$ & $\begin{array}{l}\operatorname{aadAl}(3), \operatorname{aadA2}(1), \operatorname{aadB}(3), \operatorname{bla}_{\mathrm{PSE}-1}(2), \operatorname{bla}_{\mathrm{TEM}}(3), \operatorname{dfrA12}(2), \operatorname{sull}(3), \operatorname{sul3}(2), \\
\operatorname{tet} A(3)\end{array}$ \\
\hline AMP, CHP, GEN, SPC, STR, SUL, TET & $6(3.3)$ & 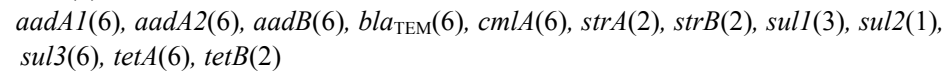 \\
\hline AMP, CHP, SPC, STR, SUL, TET, TRI & $11(6.0)$ & $\begin{array}{l}\operatorname{aadA1}(10), \operatorname{aadA} 2(1), \operatorname{bla} a_{\mathrm{PSE}-1}(5), \operatorname{bla} a_{\mathrm{TEM}}(9), \operatorname{cml} A(8), \operatorname{dfr} A 10(1), d f r A 12(5), \operatorname{str} A(9), \\
\operatorname{str} B(9), \operatorname{sull}(6), \operatorname{sul}(3), \operatorname{sul}(9), \operatorname{tet} A(8), \operatorname{tet} B(3)\end{array}$ \\
\hline AMP, GEN, SPC, STR, SUL, TET, TRI & $2(1.1)$ & $\begin{array}{l}\operatorname{aadAl}(2), \operatorname{aadA2}(2), \operatorname{aadB}(1), \operatorname{bla}_{\mathrm{PSE}-1}(1), \operatorname{bla} a_{\mathrm{TEM}}(1), \operatorname{dfr} A 12(2), \operatorname{str} A(1), \operatorname{str} B(1), \\
\operatorname{sul1}(2), \operatorname{sul}(1), \operatorname{tet} A(2)\end{array}$ \\
\hline $\begin{array}{l}\text { AMP, CHP, GEN, SPC, STR, SUL, } \\
\text { TET, TRI }\end{array}$ & $6(3.3)$ & $\begin{array}{l}\operatorname{aadAl}(6), \operatorname{aadA2}(6), \operatorname{aad} B(4), \operatorname{bla}_{\mathrm{PSE}-1}(4), \operatorname{bla}_{\mathrm{TEM}}(6), \operatorname{cml} A(4), \operatorname{dfr} A 12(6), \operatorname{str} A(2), \\
\operatorname{str} B(2), \operatorname{sul}(6), \operatorname{sul} 2(5), \operatorname{sul} 3(5), \operatorname{tet} A(6), \operatorname{tet} B(1)\end{array}$ \\
\hline
\end{tabular}

a) Abbreviations: AMP, ampicillin; CHP, chloramphenicol; CIP, ciprofloxacin, GEN, gentamicin; SPC, spectinomycin, STR, streptomycin, SUL, sulfamethoxazole, TET, tetracycline; TRI, trimethoprim.

a Thr-57-Ser substitution in ParC was found in the other serovar Senftenberg isolate. No mutations were observed in the QRDRs of the ciprofloxacin sensitive control.

Antimicrobial resistance patterns and resistance gene profiles are shown in Table 3. All the strains harboring more than one resistance gene were resistant to three or more antimicrobials. Statistical analysis showed that there were significant associations between being resistance to an antimicrobial agent and having resistance gene for all genes $(\mathrm{p}<0.01)$ except $c a t B$ and $d f r A 10$, which were not significantly associated with being resistance to chloramphenicol and trimethoprim respectively $(\mathrm{p}=0.051)$. Having more than one gene conferring resistance to a single agent was significantly associated with increasing MIC level and proportion of resistance isolates $(\mathrm{p}<0.01)$. In addition, having genes conferring resistance to multiple drugs was significantly associated with being multiresistance $(p<0.01)$. Twenty-two percent of the Salmonella isolates were nega- 
tive to all the resistance genes tested, even through most of these isolates were resistant to at least one antimicrobial agent (data not shown).

\section{DISCUSSION}

Our findings showed that most of the antimicrobial resistance genes tested were found at high rates, indicating that these genes play an important role in conferring resistance among the Salmonella isolates in this study. This data also confirmed the wide diversity of mechanisms mediating antimicrobial resistance in different Salmonella serovars of animal origins. These investigated resistance genes were previously observed at high rate in Salmonella from humans, food animals and food products $[1,4,19]$.

As class 1 integrons were previously found to be prevalent in the resistant Salmonella isolates of our collection [15], integrons of either class 2 and 3 were not identified in this study. Several reports showed that class 1 integrons were more frequently observed than class 2 integrons [19, 28] and the rarely described class 3 integrons are to date not identified in this pathogen [28]. These results once again highlight the important role of class 1 integrons in dissemination of antimicrobial resistance.

Resistance to quinolones in Salmonella is usually a result of a single point mutation in the QRDR of the gyrA gene (nucleotides 67 to 122) [12], which is consistent with the results in this study. The mutation Ser-83-Phe and Thr-57Ser had been previously described $[9,10,17]$. However, a ciprofloxacin-resistant strain did not have mutations in gyrA, gyrB, parC, and parE. The possible resistance mechanisms for the isolates could be decreased permeability of the outer membrane, an active efflux $[5,12]$ or the existence of as yet unknown additional resistance mechanisms. These results warrant further investigation.

Up to $53 \%$ of the isolates harbored 2 or 3 different resistant genes for the same antimicrobials, such as sull, sul2 and $s u l 3$ for sulphonamide resistance or $d f r A 10$ and $d f r A 12$ for trimethoprim resistance. These included all of the strains that contained class 1 integrons. For these class 1 integronspositive strains, it is possible that one resistance gene was associated with the integrons and gene clusters on SGI1 variants, but the other gene(s) were located in a plasmid [26]. However, some of the class 1 integrons-negative strains also carried more than one resistance gene for the same antimicrobial resistance. Further studies to characterize plasmids and elucidate the alternative possibilities are required.

The prevalence of chloramphenicol resistance and the existence of the chloramphenicol resistance gene (s) among $E$. coli isolates has been demonstrated $[2,27]$ even through the antibiotic has been prohibited for using in domestic animals. This is in agreement of the results in our study, which the $\mathrm{cmlA}$ gene was found in the majority of chloramphenicol-resistant isolates. In our previous study, the $\mathrm{cmlA}$ gene in 7 chloramphenicol-resistant Salmonella strains was shown to be part of gene cassettes in class 1 integrons with unusual 3' conserved segment linked to the qacH-sul3 domain and the common resistance genes in the gene cassettes included $a a d A 1$ and $a a d A 2$, and $q a c H$ [7]. This closegenetic linkage to other resistance genes could be a mechanism for maintenance and co-selection of chloramphenicol resistance in Salmonella in the absence of chloramphenicol selection pressure [2, 27].

The present study demonstrated good correlations between the presence of antimicrobial resistance genes and corresponding resistance phenotype suggesting that these resistance genes were usually expressed when present. For each antimicrobial agent, the MIC level of the Salmonella isolates carrying more than one corresponding-resistance gene was higher than that of the strains with only a single gene (data not shown), indicating the synergy among the genes in mediating resistance. However, these results did not rule out that other resistance genes not tested in this study are also present. Several resistant-Salmonella isolates did not carry the investigated resistance genes, indicating that there must be other mechanisms, which contributed to such resistance and were not tested in this study e.g. active efflux and other inactivating enzymes.

In conclusion, the results in this study provide useful information regarding the dissemination of antimicrobial resistance genes among a wide range of the resistant Salmonella serovars confirming an important role of food animals in the spread of antimicrobial resistance. Antimicrobialresistant bacteria and their resistance determinants are considered hazards in antimicrobial resistance risk assessment; therefore, the information obtained could be used as part of risk analysis of the distribution and development of antimicrobial resistance. However, data regarding the epidemiological situations of antimicrobial resistance and mechanism basis of resistance is still limited especially in most developing countries. Continued monitoring of antimicrobial resistance in a huge strain collection of Salmonella along the food chain is required so that comparison of antimicrobial resistance from the different origins could be effectively performed. Guidelines on the prudent use of antimicrobials in animal production should be developed and readily accessible. The guidelines should base on existing regulation, resistance situation and human health concerns and include recommendations on a rational antimicrobial treatment of choice.

ACKNOWLEDGMENTS. We thank S. Khemtong and W. Wannaprasat for technical assistance. P. Pathanasophon, NIAH was acknowledged for strain providing. This study was supported by Chulalongkorn University Rachadapisaksompoch Endowment Fund in the fiscal year 2007 to R. Chuachuen. It is supported in part by the Thailand Research Fund to P. Padungtod

\section{REFERENCES}

1. Aarestrup, F. M., Lertworapreecha, M., Evans, M. C., Bangtrakulnonth, A., Chalermchaikit, T., Hendriksen, R. S. and Wege- 
ner, H. C. 2003. Antimicrobial susceptibility and occurrence of resistance genes among Salmonella enterica serovar Weltevreden from different countries. J. Antimicrob. Chemother. 52: 715-718.

2. Bischoff, K. M., White, D. G., Hume, M. E., Poole, T. L. and Nisbet, D. J. 2005. The chloramphenicol resistance gene $\mathrm{cmlA}$ is disseminated on transferable plasmids that confer multipledrug resistance in swine Escherichia coli. FEMS Microbiol. Lett. 243: 285-291.

3. Butaye, P., Michael, G. B., Schwarz, S., Barrett, T. J., Brisabois, A. and White, D. G. 2006. The clonal spread of multidrugresistant non-typhi Salmonella serotypes. Microbes. Infect. 8: 1891-1897.

4. Chen, S., Zhao, S., White, D. G., Schroeder, C. M., Lu, R., Yang, H., McDermott, P. F., Ayers, S. and Meng, J. 2004. Characterization of multiple-antimicrobial-resistant salmonella serovars isolated from retail meats. Appl. Environ. Microbiol. 70: $1-7$.

5. Choi, S. H., Woo, J. H., Lee, J. E., Park, S. J., Choo, E. J., Kwak, Y. G., Kim, M. N., Choi, M. S., Lee, N. Y., Lee, B. K., Kim, N. J., Jeong, J. Y., Ryu, J. and Kim, Y. S. 2005. Increasing incidence of quinolone resistance in human non-typhoid Salmonella enterica isolates in Korea and mechanisms involved in quinolone resistance. J. Antimicrob. Chemother. 56: $1111-1114$.

6. Chuanchuen, R., Khemtong, S. and Padungtod, P. 2007. Occurrence of qacE/qacED1 genes and their correlation with class 1 integrons in Salmonella enterica isolates from poultry and swine. Southeast Asian J. Trop. Med. Public. Health 38: 855-862.

7. Chuanchuen, R., Koowatananukul, C. and Khemtong, S. 2008. Characterization of class 1 integrons with unusual 3' conserved region from Salmonella enterica isolates. Southeast Asian J. Trop Med Public Health 39: 419-424.

8. Chuanchuen, R., Pathanasophon, P., Khemtong, S., Wannaprasat, W. and Padungtod, P. 2008. Susceptibilities to antimicrobials and disinfectants in Salmonella enterica isolates obtained from poultry and swine in Thailand. J. Ved. Med. Sci. 70: 595501.

9. Eaves, D. J., Liebana, E., Woodward, M. J. and Piddock, L. J. 2002. Detection of gyrA mutations in quinolone-resistant $\mathrm{Sal}$ monella enterica by denaturing high-performance liquid chromatography. J. Clin. Microbiol. 40: 4121-4125.

10. Escribano, I., Rodriguez, J. C. and Royo, G. 2004. Mutations in the gyrA gene in Salmonella enterica clinical isolates with decreased ciprofloxacin susceptibility. Int. J. Antimicrob. Agents 24: 300-303.

11. Gebreyes, W. A. and Altier, C. 2002. Molecular characterization of multidrug-resistant Salmonella enterica subsp. enterica serovar Typhimurium isolates from swine. J. Clin. Microbiol. 40: $2813-2822$.

12. Guerra, B., Malorny, B., Schroeter, A. and Helmuth, R. 2003. Multiple resistance mechanisms in fluoroquinolone-resistant Salmonella isolates from Germany. Antimicrob. Agents Chemother. 47: 2059.

13. Hanson, R., Kaneene, J. B., Padungtod, P., Hirokawa, K. and Zeno, C. 2002. Prevalence of Salmonella and E. coli, and their resistance to antimicrobial agents, in farming communities in northern Thailand. Southeast Asian J. Trop. Med. Public Health 33 (Suppl.)3: 120-126.

14. ISO. 2002. Microbiology of food and animal feeding stuffsHorizontal method for the detection of Salmonella spp: ISO6579, 4th ed, vol. Reference number ISO 6579: 2002(E).
15. Khemtong, S. and Chuanchuen, R. 2008. Class 1 Integrons and Salmonella Genomic Island 1 among Salmonella enterica Isolated from Poultry and Swine. Microbe. Drug Resist. 14: 6570 .

16. Levesque, C., Piche, L., Larose, C. and Roy, P. H. 1995. PCR mapping of integrons reveals several novel combinations of resistance genes. Antimicrob. Agents Chemother. 39: 185-191.

17. Ling, J. M., Chan, E. W., Lam, A. W. and Cheng, A. F. 2003. Mutations in topoisomerase genes of fluoroquinolone-resistant salmonellae in Hong Kong. Antimicrob. Agents Chemother. 47: 3567-3573.

18. McEwen, S. A. and Singer, R. S. 2006. Stakeholder position paper: the need for antimicrobial use data for risk assessment. Prev. Vet. Med. 73: 169-176.

19. Miko, A., Pries, K., Schroeter, A. and Helmuth, R. 2005. Molecular mechanisms of resistance in multidrug-resistant serovars of Salmonella enterica isolated from foods in Germany. J. Antimicrob. Chemother. 56: 1025-1033.

20. NCCLS. 2002. Performance Standards for Antimicrobial Disk and Dilution Susceptibility Tests for Bacteria Isolated from Animals; Approved Standard-Second Edition. NCCLS document M31-A2. NCCLS, PA, U.S.A.

21. NCCLS. 1998. Performance standards for antimicrobial susceptibility testing. Eight informational supplement. NCCLS Document M100-S8. NCCLS, PA, U.S.A.

22. O'Mahony, R., Saugy, M., Leonard, N., Drudy, D., Bradshaw, B., Egan, J., Whyte, P., O'Mahony, M., Wall, P. and Fanning, S. 2005. Antimicrobial resistance in isolates of Salmonella spp. from pigs and the characterization of an S. Infantis gene cassette. Foodborne Pathog. Dis. 2: 274-281.

23. Padungtod, P. and Kaneene, J. B. 2006. Salmonella in food animals and humans in northern Thailand. Int. J. Food Microbiol. 108: $346-354$.

24. Padungtod, P., Kaneene, J. B., Hanson, R., Morita, Y. and Boonmar, S. 2006. Antimicrobial resistance in Campylobacter isolated from food animals and humans in northern Thailand. FEMS Immunol. Med. Microbiol. 47: 217-225.

25. Popoff, M. and LeMinor, L. 1992. Antigenic formulas of the Salmonella Serovars, 7th ed. Institut Pastur, Paris.

26. Randall, L. P., Cooles, S. W., Osborn, M. K., Piddock, L. J., and Woodward, M. J. 2004. Antimicrobial resistance genes, integrons and multiple antimicrobial resistance in thirty-five serotypes of Salmonella enterica isolated from humans and animals in the UK. J. Antimicrob. Chemother. 53: 208-216.

27. Sunde, M. and Norstrom, M. 2006. The prevalence of, associations between and conjugal transfer of antimicrobial resistance genes in Escherichia coli isolated from Norwegian meat and meat products. J. Antimicrob. Chemother. 58: 741-747.

28. van Essen-Zandbergen, A., Smith, H., Veldman, K. and Mevius, D. 2007. Occurrence and characteristics of class 1, 2 and 3 integrons in Escherichia coli, Salmonella and Campylobacter spp. in the Netherlands. J. Antimicrob. Chemother. 59: 746-750.

29. Xu, H., Davies, J. and Miao, V. 2007. Molecular characterization of class 3 integrons from Delftia spp. J. Bacteriol. 189: 6276-6283.

30. Yang, S. J., Park, K. Y., Kim, S. H., No, K. M., Besser, T. E., Yoo, H. S., Kim, S. H., Lee, B. K. and Park, Y. H. 2002. Antimicrobial resistance in Salmonella enterica serovars Enteritidis and Typhimurium isolated from animals in Korea: comparison of phenotypic and genotypic resistance characterization. Vet. Microbiol. 86: 295-301. 